\title{
Modelling star counts in the Monoceros stream and the Galactic anti-centre
}

\author{
P. L. Hammersley ${ }^{1,2,3}$ and M. López-Corredoira ${ }^{1,2}$ \\ 1 Instituto de Astrofísica de Canarias, 38200 La Laguna, Tenerife, Spain \\ e-mail: [peter.hammersley; martinlc]@iac.es \\ 2 Departamento de Astrofísica, Universidad de La Laguna, La Laguna, Tenerife, Spain \\ ${ }^{3}$ European Southern Observatory, Karl-Schwarzschild-Strasse 2, Garching 85748, Germany
}

Received 3 November 2009 / Accepted 8 November 2010

\section{ABSTRACT}

\begin{abstract}
Context. There is a continuing debate as to the form of the outer disc of the Milky Way galaxy, which has important implications for understanding the Galaxy's formation. Stars are known to exist at a galacto-centric distance of at least 20 kpc. However, there is much debate as to whether these stars can be explained as part of the disc or whether another extragalactic structure, the so-called Monoceros ring/stream, is required

Aims. We examine the outer disc of the Galaxy toward the anti-centre to determine whether the star counts can be explained by the thin and thick discs alone.

Methods. By using Sloan star counts and extracting the late F and early G dwarfs, it is possible to directly determine the density of stars out to a galacto-centric distance of about $25 \mathrm{kpc}$. These are then compared with a simple flared-disc model.

Results. A flared-disc model is shown to reproduce the counts along the lines of sight examined, if the thick disc does not have a sharp cut off. The flare starts at a Galacto-centric radius of $16 \mathrm{kpc}$ and has a scale length of $4.5 \pm 1.5 \mathrm{kpc}$

Conclusions. Whilst the interpretation of the counts in terms of a ring/stream cannot be definitely discounted, it does not appear to be necessary, at least along the lines of sight examined towards the anti centre.
\end{abstract}

Key words. Galaxy: structure - Galaxy: disk - Galaxy: stellar content

\section{Introduction}

The position of the Sun within the Galactic plane has made understanding the true form of the Galaxy difficult. In the past ten years the situation has improved, however, with the advent of deep large-area surveys. Surveys in the near IR, such as 2MASS and UKIDSS, or in the visible such as the Sloan Digital Sky Survey (SDSS, Newberg et al. 2002), have allowed a more precise modelling of the large-scale structures of our Galaxy.

It is generally accepted that there is both a thin and a thick stellar disc. The stellar density of the thin disc is exponential in height above the Galactic plane and radial distance. In the vicinity of the Sun it has a scale length of about $2.4 \mathrm{kpc}$; however, the scale height depends on the source type. The older sources have a scale height of around $185 \mathrm{pc}$, but it is considerably less than this for the younger sources. The density of the thick disc (Gilmore \& Reid 1983) is also exponential but with a larger scale length and scale height (Bilir et al. 2008), although it has less than a tenth of the density of the thin disc on the plane in the vicinity of the Sun.

Of particular importance to our understanding of the Galaxy and its formation, is to determine what happens towards the outer edge of the disc. Using visible images from the CFHT, Robin et al. (1992) suggested that Galaxy has a cut of at galacto-centric radius $(R)$ of $13.5 \mathrm{kpc}$ when looking at $l=179^{\circ} b=-2.5^{\circ}$, because the stellar density drops rapidly beyond that point. Using 2MASS data, López-Corredoira et al. (2002) suggests that the drop in stellar density near the plane was not caused by a cut off, but rather by a flare in the disc. They conclude that the disc did not have a well-defined cut off to at least $15 \mathrm{kpc}$.
The most recent deep surveys such as the SDSS clearly show that there are stars out to at least $R=20 \mathrm{kpc}$. Their distribution, however, is not consistent with a simple exponential disc particularly when looking 15 to 30 degrees away from the plane. Some authors (Momany et al. 2006) suggest these can be explained by a flare of the disc. Many other authors, however, have preferred to attribute these sources to rings or streams beyond the edge of the disc. In particular, the sources being studied here have been attributed to the so-called Monoceros ring/stream (Newberg et al. 2002; Rocha-Pinto et al. 2003; Conn et al. 2005, 2007, 2008). This has been associated to the remnants of a dwarf galaxy that was cannibalised by the Milky Way, and its progenitor was associated to the Canis Major over-density (Martin et al. 2004; Martínez-Delgado et al. 2005; Bellazzini et al. 2006; Butler et al. 2007; de Jong et al. 2007; Conn et al. 2007). This ring runs approximately parallel to the Galactic plane, in the latitude range $10<|b|<35$ deg and over most of the second and third quadrants.

That stellar streams exist is well known, and the one produced by the Sagittarius dwarf galaxy (Ibata et al. 1994) is probably the best studied stream associated with the Milky Way. Its form and high angle to the Galactic plane makes confusion with a Galactic component highly unlikely. However, the proposed Monoceros stream is a very different feature to the Sagittarius stream as it runs parallel to the Galactic plane. Furthermore, whilst the Sagittarius stream is clearly a dwarf galaxy, the progenitor of Monoceros in the over-density in Canis Major has also been questioned, as other authors have explained the excess star as an effect of the warped+flared disc of the 
Milky Way (Momany et al. 2004, 2006; López-Corredoira 2006; López-Corredoira et al. 2007).

In this paper we examine whether by using a simple flareddisc model as suggested by Momany et al. (2006), we can reproduce the form of the deep star counts seen 15 to 30 degrees off the plane in Galactic anti-centre region, without requiring the presence of extra-galactic streams.

\section{The method}

For this work we wish to count sources at $R<25 \mathrm{kpc}$ towards the Galactic anti-centre and between 11 and 31 degrees off the plane. By far the simplest method of determining the stellar density along a line of sight in the disc is by isolating a group of stars with the same colour and absolute magnitude within a colourmagnitude diagram. This allows the luminosity function to be replaced by a constant in the stellar statistics equation:

$$
\begin{aligned}
A(m) & \equiv \frac{\mathrm{d} N(m)}{\mathrm{d} m}=\frac{\ln 10}{5} \omega \rho[r(m)] r(m)^{3}, \\
r(m) & =10^{[m-M+5] / 5},
\end{aligned}
$$

where $\omega$ is the area of the solid angle in radians and $r$ the distance in parsecs. The differential star counts for each line of sight, $A(m)$, can be immediately converted into density $\rho(r)$.

In the near IR, the red clump giants have been successfully used as standard candles, particularly when looking in the inner Galaxy (López-Corredoira et al. 2002). However, the red clump stars in the outer disc at the distance of interest would appear at $m_{k}=15$, so the local dwarfs with the same colour would completely dominate the counts at this magnitude (López-Corredoira et al. 2002).

As the areas of interest all lie well off the plane, then the extinction will be low (typically under 1 magnitude). Therefore, it is possible to use visible counts. An examination of the HR diagram shows that when the extinction is low, the late $F$ and early $\mathrm{G}$ dwarfs can be isolated using colour with only minimal contamination from other sources with the same colour but different absolute magnitudes (sub-dwarfs, galacto-centric radius giants, etc.). For this work we have selected the source between F8V and G5V. This gives a range of $g-r$ of 0.36 to 0.49 and a range of absolute magnitudes $M_{g}=4.2$ to 5.4 , which makes the sources approximately $m_{g} \sim 20$ at the distance of interest. By having a range of absolute magnitudes, then when the counts are converted to density vs distance, there is some smoothing. This is not included in a model, which assumes a single absolute magnitude. Although the selection used here is not as well defined as the red clump stars in the near IR, it still has a sufficiently narrow range in absolute magnitude that the smoothing has little effect and the above approximation remains valid. There will, however, be enough stars detected in the outer Galaxy to give meaningful statistics, which would not be the case if a narrower range in absolute magnitudes were used. Earlier sources have not been included, since these sources would belong to a younger population with a far smaller scale height, and the absolute magnitude also changes far more rapidly with colour. Later sources would have significant giant contamination, and the absolute magnitude again changes more rapidly with colour.

The areas selected for this work lie between 10 and 30 degrees from the plane and are within 40 degrees of the anti-centre. We have not extended the range of longitudes because of the Galactic warp. The warp means that the position of the Galactic plane varies with position in the Galaxy, and along the line of sight. Although the effect of warp is strongest towards the outer
Table 1. Lines of sight used.

\begin{tabular}{cccc}
\hline \hline$l\left(^{\circ}\right)$ & $b\left(^{\circ}\right)$ & Area $\left(\mathrm{deg}^{2}\right)$ & Extinction $E(B-V)$ \\
\hline 150 & 15 & 1.94 & 0.25 \\
180 & 11 & 0.45 & 0.241 \\
183 & 21 & 1.22 & 0.054 \\
183 & 31 & 3.27 & 0.052 \\
223 & 20 & 1.00 & 0.035 \\
\hline
\end{tabular}

edge of the disc, where the plane can be over a kpc away from the expected position with no warp, it can be clearly seen in the counts within a few kpc of the Sun (Hammersley et al. 1995; López-Corredoira et al. 2002; Momany et al. 2006). The models of the warp are normally simple, based on tilted rings, and in general these do provide a good representation of the star counts. However, the models are harder to test in the very outer disc, and a small error in the model can lead to a significant error in the predicted position of the plane, hence in the predicted densities. Therefore, we limited the regions used for this work to those where the effect of the warp should be small and so can be ignored. It should be noted, however, that, whilst it will be possible to ascertain whether a flare can reproduce the star counts seen in the outer disc, it will not be possible to accurately determine all of the parameters. When large-scale deep star counts in the outer disc become available it will be possible to model the warp more accurately. Until then, however, great care must be taken when comparing models with counts in the outer disc.

A second reason for limiting the longitude is that the distance to the feature increases rapidly with distance from the anticentre, making the sources fainter and appear closer to the plane so that there is less contrast with the other disc sources.

\section{The data}

The source densities in these regions will be very low, requiring square degrees of sky to be covered to provide sufficient counts to give reasonable statistics. Therefore, the data has been taken from the SDSS release DR7 (Abazajian et al. 2009) with regions $\sim 1$ square degree. As the regions are all well off the plane, it has been assumed that the extinction is local, so all of the magnitudes have been corrected for extinction using the Galactic extinction model of Schlegel et al. (1998). Furthermore, the extinction is relatively small and a small residual error will not significantly affect the results, although it would make the sources appear at the wrong distance. Table 1 shows the position and assumed extinctions. The positions $l=183^{\circ}, b=21^{\circ}$ and $l=220^{\circ}$ were used by Newberg et al. (2002), and the position at $l=150^{\circ}$ was used by Conn et al. (2005), to support their "Monoceros stream" hypothesis.

\section{Results}

Figure 1 shows the $g$ vs. $g-r$ colour magnitude diagram for the region $l=183^{\circ}, b=21^{\circ}$ with the sources of interest marked. It can be seen they are in a reasonably uncluttered part of the diagram allowing them to be isolated. Furthermore, there are no "holes" in the area of interest indicating that there are no areas of high extinction. Figure 2 shows the differential counts for the selected colour along with the Besançon model (Robin et al. 2003) for these stars. This model is widely used and generally 


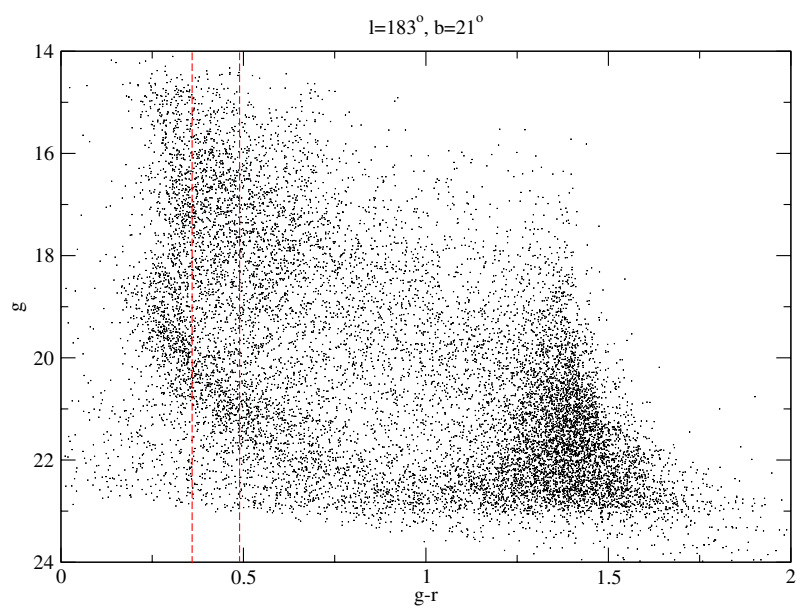

Fig. 1. The CMD for the region $l=183^{\circ}, b=21^{\circ}$

accurately predicts the counts over wide areas of the sky. Here we have only run the model for the stars between F9V and G4V. The model was run with no extinction and predicted counts in $V$ which were then converted to $g$ using the $g-V$ colour for these stars. The Poissonian error bars from the counts in each magnitude bin is also shown.

The counts have two clear peaks, one at about $m_{g}=17$ and a second at $m_{g}=20.7$ with a minimum at $m_{g}=19.5$ (see Fig. 2). This is seen most clearly at $b=21^{\circ}$, and $b=31^{\circ}$; however, even in the region $l=180^{\circ}, b=11^{\circ}$ there is some evidence for excess counts. The Besançon model does give a good fit to the data for $m_{g}<18$; however, at $m_{g}=20$ there is a sharp rise in the counts which is not predicted by the model. The peak corresponds with a distance of about $15 \mathrm{kpc}$ from the Sun or $R=23 \mathrm{kpc}$. This second peak cannot be caused by the halo, which is included in the model Besançon since the peak for these counts is predicted to start at $m_{g} \approx 23$ and reach a peak at about $m_{g}=25$. Therefore, there are two possible alternatives: this is part of the disc or an extra galactic component, such as a stream beyond the disc of the Galaxy.

\section{Analysis}

These star counts cannot be produced by a simple exponential disc, even when including both the thin and thick discs, as such a model cannot produce this second bump (see Fig. 2). Even if there were no-cut off this would only slightly affect the counts. However, a flared disc with no cut-off may produce the second peak along specific lines of sight, as it would on average distribute stars away from the plane. To explore this possibility, Eq. (1) was used directly to determine the stellar density with distance assuming that the luminosity function can be replaced by a delta function with $M_{g}=4.80$. Figure 3 shows the density with galacto-centric radius for the lines of sight used. It is notable that the stellar density is continuously decreasing, although it does change slope, without significant gaps or bumps, suggesting that it may be explained by only a single continuous Galactic structure and does not require two separate structures. Hence the second bump in Fig. 2 is an effect of the shallower slope in the stellar density being multiplied by the factor $r^{3}$ (see Eq. (1)).

To determine the effect of a flare, a simple model was built that contains a thin disc(scale height $h_{z, \text { thin, } \odot}=186 \mathrm{pc}$ and scale length $h_{R \text {,thin }}=2400 \mathrm{pc}$ ), and a thick disc (scale height $h_{z, \text { thick, } \odot}=631 \mathrm{pc}$ and scale length $h_{R \text {,thick }}=3500 \mathrm{pc}$ ) was
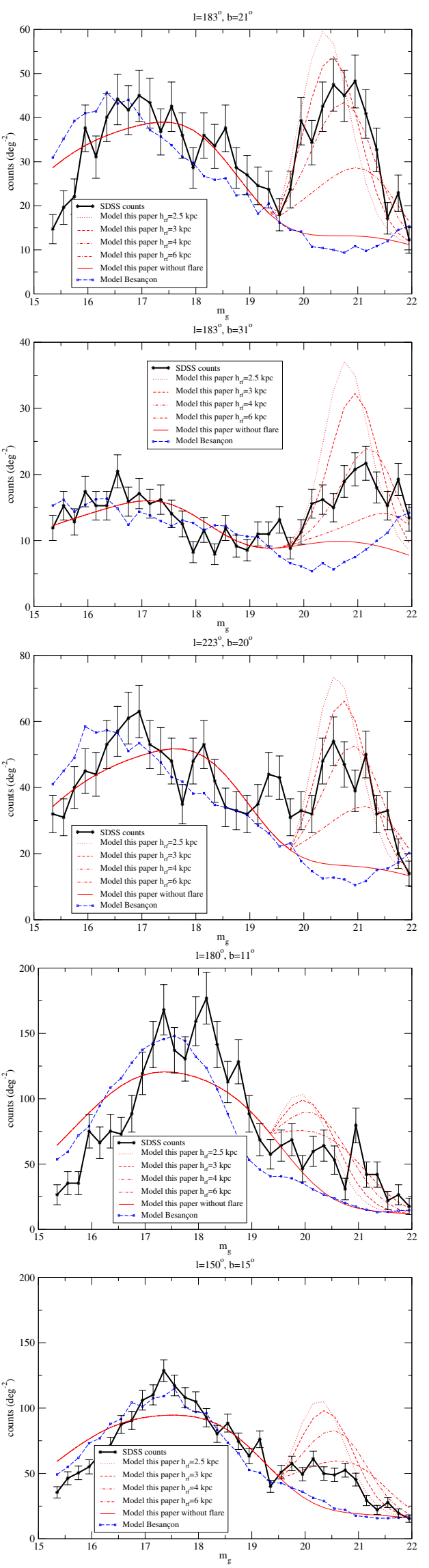

Fig. 2. The differential star counts with the error bars in five regions towards the anti centre. Along with the counts, the predictions of the Besançon model and a simple flared or non-flared disc model are also shown. 

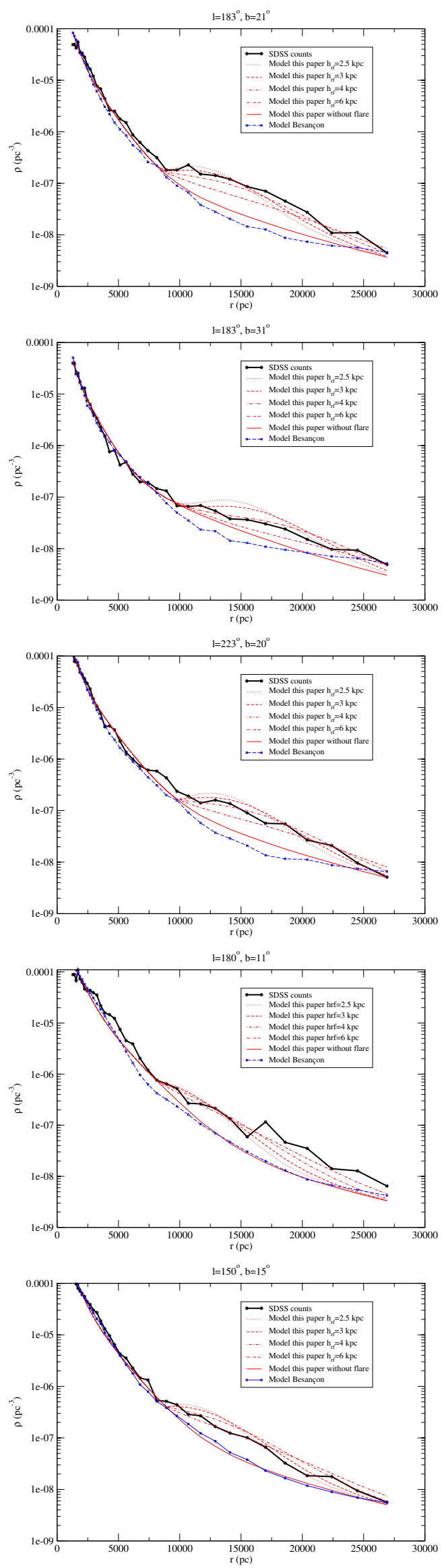

Fig. 3. The stellar density with $R$ along five lines of sight towards the anti centre. Along with the counts the predictions of the Besançon model and a simple flared or non-flared disc model are also shown. assumed with the density of the thick disc being $9 \%$ of that of the thin disc on the plane at the solar radius (as observed at high latitude with SDSS; Bilir et al. 2008). The halo counts of the model by Bilir et al. (2008) were also included, although they give a negligible contribution at these magnitudes. It was assumed that the flare increases the scale height of both the thin and thick discs beyond a certain galacto-centric radius, and that the size of the flare increases exponentially. It should be noted, however, that the thin disc only provides a significant contribution to the counts where the line of sight is within a few hundred pc of the plane. The flare begins at about $R=16 \mathrm{kpc}$, at which distance the lines of sight are over a kpc from the plane, so the contribution of the thin disc to the counts is negligible.where the flare is important. Therefore, this paper only analyses the effect of the flare on the thick disc. Lines of sight much closer to the plane would be required to analyse the effect of the flare on the thin disc, and only then can any relationship between the flare in the thin and thick discs be determined.

It was also assumed that the total number of stars at a galacto-centric radius followed a simple exponential model. If this is not done, then the large scale-height caused by the flare would lead to more stars in total at that galacto-centric radius.

The variation in stellar density is given by

$$
\begin{aligned}
& \rho=\rho_{\text {thin }}+\rho_{\text {thick }}+\rho_{\text {halo }}, \\
& \rho_{\text {thin }}=A\left(\frac{h_{z \text {,thin } \odot}}{h_{z \text {,thin }}(R)}\right) \exp \left(-\frac{R-R_{\odot}}{h_{R, \text { thin }}}\right) \exp \left(-\frac{|z|}{h_{z, \text { thin }}(R)}\right) \\
& \rho_{\text {thick }}=0.09 A\left(\frac{h_{z, \text { thick } \odot}}{h_{z, \text { thick }}(R)}\right) \exp \left(-\frac{R-R_{\odot}}{h_{R, \text { thick }}}\right) \exp \left(-\frac{|z|}{h_{z, \text { thick }}(R)}\right) \\
& \rho_{\text {halo }}=1.4 \times 10^{-3} A \frac{\exp \left[10.093\left(1-\left(\frac{R_{s p}}{R_{\odot}}\right)^{1 / 4}\right)\right]}{\left(R_{\mathrm{sp}} / R_{\odot}\right)^{7 / 8}} ; \\
& h_{z \text {,thin/thick }}(R)=\left\{\begin{array}{ll}
h_{z, \text { thin/thick } \odot \odot}, & R \leq 16 \mathrm{kpc} \\
h_{z, \text { thin/thick }, \odot} \exp \left(\frac{R-16 \mathrm{kpc}}{h_{\mathrm{rf}}}\right), & R>16 \mathrm{kpc}
\end{array}\right\} \\
& R_{\mathrm{sp}}=\sqrt{R^{2}+2.52 z^{2} .}
\end{aligned}
$$

This is, therefore, the standard thin and thick exponential disc model, but with a flare starting at a specific galacto-centric radius. There are only two free parameters, the position of the start of the flare and the scale length of the flare

It should be noted that the aim is not to try to fit the model to the data but to demonstrate that a simple model does reproduce the counts. Effects such as errors in the extinction calculation, residual effects of the warp, and the expected none axi-symmetry of the outer disc would be require a far more complex model and a far broader sky coverage, in particular closer to the plane.

In Figs. 2 and 3 the effects of various flares starting at a galacto-centric radius of $16 \mathrm{kpc}$ are shown. For Fig. 2 the amplitude $A$ has been normalised to the measured counts between $m_{g}$ of 16 and 19, which is well before the flare starts. In Fig. 3, however, the density has been normalised to the measured $\int_{0}^{\infty} \rho(r) \mathrm{d} r$.

Figure 4 takes the densities and determines by how much the disc would need to be flared at each galacto-centric radius to give the determined density. At the radius of the Sun, the relative scale height is 1 , and it remains constant until an $R$ of $16 \mathrm{kpc}$, at which point the scale height increases rapidly. It is true that the flare is not identical in each case, however, they are very similar, with a typical flare scale length of $4.5 \pm 1.5 \mathrm{kpc}$ (Fig. 4). There 


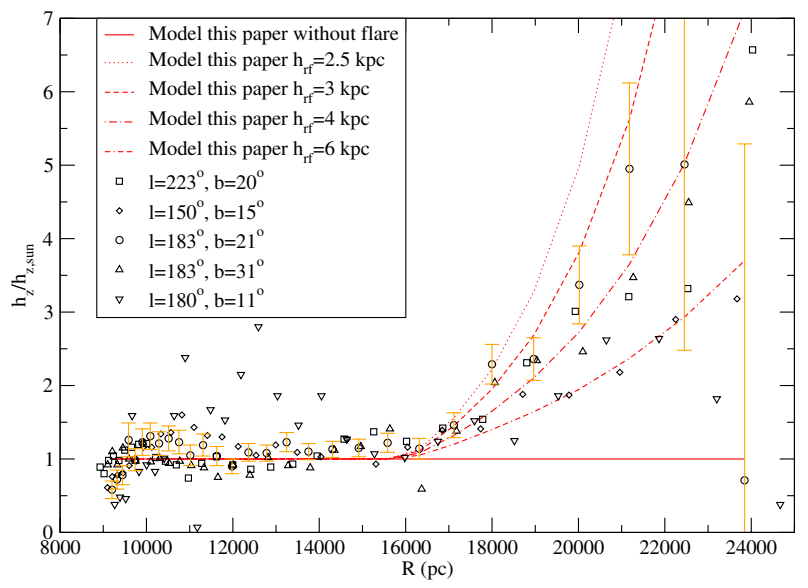

Fig. 4. The proportional flare in the scale height required to give the counts at each magnitude along each line of sight. The error bars from the number of sources detected in each magnitude bin are shown for one region.

are, however, a number of effects that have not been taken into account.

- The warp has been ignored here, but would mean that the average plane position would be above $b=0$ in the $l=150^{\circ}$ region and below $b=0$ at $220^{\circ}$. In the region 10-18 degrees from the plane the counts from the flare increases rapidly, and so a small error in the position of the plane would significantly reduce the counts at $l=150^{\circ} \mathrm{b}=15$.

- The simple model used here assumes a circularly symmetric outer Galaxy. As we are dealing here with the thick disc at 6 to 7 scale lengths from the Galactic centre, then it would lead to a $50 \%$ change in counts if there were a $10 \%$ change in scale length with Galactic longitude.

- The model used here assumes that the parameters of the flare do not depend on Galatic longitude.

- The extinction model used here is very simplistic.

Finally it should be noted that the counts for most distant points are very low leading to a large scatter. When many more regions become available, it will be possible to quantify these effects and so refine the results.

These results do show that the variation in the number of stars with galacto-centric distance is consistent with an exponential law out to over $20 \mathrm{kpc}$. There is also no evidence of either a major drop in counts just before the flare starts or a sudden jump in the counts. This means that the counts in the disc can be described by a single function to beyond $20 \mathrm{kpc}$.

These plots show that the simple flare model not only predicts that at 20 degrees from the plane there should be two peaks in the star counts, but also it can reproduce the numbers of counts and form.

The alternative scenario is a ring of stars centred at $R=$ $23 \mathrm{kpc}$, which is detected for $R>16-17 \mathrm{kpc}$ (Conn et al. 2007). Since this structure would only exist in the outer Galaxy, its parameters are not constrained to fit observations in the solar neighbourhood or elsewhere in the Galaxy. This means that there is a wide range of possible parameter space that can be explored, so that by adjusting the width and density, a ring can be made to fit second peak. However, this ring must start very close to the edge of the disc. If the inner part of the stream is more than 1 or $2 \mathrm{kpc}$ from the edge of the disc, then there would be a noticeable drop in the stellar density at that point. Conversely, if it overlapped with the edge of the disc then there would be a jump in the density. Furthermore, the number of stars in the stream has to be very similar to those predicted for the disc with no cut off, otherwise none of the flared models would come close to reproducing the measured counts. Finally the vertical distribution of the counts again has to mimic the flared disc model, and this is over more that 20 degrees in latitude, which correspond to 3 to $4 \mathrm{kpc}$ at an $R$ of 15 to $20 \mathrm{kpc}$. As a result, if there is a stream it would have to have many characteristics that are identical to those predicted for a flared outer disc.

\section{Discussion}

That the disc of the Galaxy is flared is widely accepted and is particularly well studied in the HI (e.g., Kalberla et al. 2007). They also find that the flare becomes relevant at around $R=$ $16 \mathrm{kpc}$ and that the disc continues to at least $23 \mathrm{kpc}$. There may also be a dependence of the scale height on the galacto centric azimuth, as observed in the gas (Kalberla et al. 2007), and this lopsidedness could be explained by the non-axisymmetrical distribution of pressures in the outer disc (López-Corredoira \& Betancort-Rijo 2009).

By moving sources away from the plane, the result is that once the flare starts, there will be a sharp drop in the counts when compared to the predictions of a simple exponential disc for any lines of sight near the plane, whereas at higher latitudes there will be considerably more stars than predicted. The sudden loss of stars beyond about $R=14 \mathrm{kpc}$ is reported in Robin et al. (1992) when looking at $l=179^{\circ} b=-2.5^{\circ}$, and the results here show that the second peak in the differential star counts at $b=20^{\circ}$ is entirely consistent with a flare.

The stars that are in the second peak would belong to the thick disc, since they are typically 3 to $4 \mathrm{kpc}$ above the plane and are at two to three times the galacto-centric radius of the Sun. Their metallicity has been measured to be an average of $[\mathrm{Fe} / \mathrm{H}]=-0.96$, with an rms scatter of only $\sim 0.15$ dex (Ivezić et al. 2008), which is compatible with the metallicity of the outer thick disc (López-Corredoira et al. 2007, Fig. 3).

The flare of the thick disc is important for understanding its origin, as current theories suggest that thick discs caused by mergers will always flare. Bournaud et al. (2009) find that if a flare were caused by minor mergers, then there would be no region of the thick disc with a constant scale height. The analysis presented here is not sensitive to changes in the scaleheight of thick disc closer to the solar circle, since the counts for $R<16 \mathrm{kpc}$ along the lines of site used are dominated by the thin disc. An analysis of the thick disc flare in the whole range of galactocentric distances would be necessary to see whether the mechanism proposed by Bournaud et al. ( 2009) is applicable here.

\section{Conclusions}

Uncertainties in our knowledge of the disc makes asserting the presence of the Monoceros stream, principally because a model does not accurately reproduce the star counts, dangerous, even when using a model as good as the Besançon model. If the disc has a cut-off at $R$ of about $14 \mathrm{kpc}$, as in the Besançon model, then the ring would be needed to give the star counts detected. If, however, there is no cut-off and the thick disc instead flares, then the bulk of the stars in the regions discussed here cannot belong to a stream as there are only enough stars to be explained by the disc alone. It should be noted that this would not preclude 
there being smaller scale streams towards the anti-centre affecting smaller regions of sky.

We have shown that a flare in the thick disc would leave a very clear signature in the star counts in the outer Galaxy and that this signature is seen. We have shown that both the form and number of stars detected towards the anti-centre between 11 and 31 degrees from the plane can be readily explained by a flared thick disc without a cut-off. When a larger area of sky becomes available it will be possible to refine the outer disc and flare models and thus improve the quality of the fit, in particular nearer the plane. The presence of the extra-galactic Monoceros $\mathrm{ring} / \mathrm{stream}$ is therefore not required to explain the star counts.

Acknowledgements. M.L.C. was supported by the Ramón y Cajal Programme of the Spanish Science Ministry. Funding for the SDSS and SDSS-II has been provided by the Alfred P. Sloan Foundation, the Participating Institutions, the National Science Foundation, the US Department of Energy, the National Aeronautics and Space Administration, the Japanese Monbukagakusho, the Max Planck Society, and the Higher Education Funding Council for England. The SDSS Web Site is http://www.sdss.org/. The SDSS is managed by the Astrophysical Research Consortium for the Participating Institutions. The Participating Institutions are the American Museum of Natural History, Astrophysical Institute Potsdam, University of Basel, University of Cambridge, Case Western Reserve University, University of Chicago, Drexel University, Fermilab, the Institute for Advanced Study, the Japan Participation Group, Johns Hopkins University, the Joint Institute for Nuclear Astrophysics, the Kavli Institute for Particle Astrophysics and Cosmology, the Korean Scientist Group, the Chinese Academy of Sciences (LAMOST), Los Alamos National Laboratory, the Max-Planck-Institute for Astronomy (MPIA), the Max-PlanckInstitute for Astrophysics (MPA), New Mexico State University, Ohio State University, University of Pittsburgh, University of Portsmouth, Princeton University, the United States Naval Observatory, and the University of Washington. We would also like to thank the referee for useful comments that improved the paper.

\section{References}

Abazajian, K. N., Adelman-McCarthy, J. K., Agüeros, M. A., et al. 2009, ApJS, 182,543

Bellazzini, M., Ibata, R., Martin, N., et al. 2006, MNRAS, 366, 865 Bilir, S., Cabrera-Lavers, A., Karaali, S., et al. 2008, PASA, 25, 69 Bournaud, F., Elmegreen, B. G., \& Martig, M. 2009, ApJ, 707, L1

Butler, D. J., Martínez-Delgado, D., Rix, H.-W., Peñarrubia, J., \& de Jong, J. T. A., 2007, AJ, 133, 2274

Conn, B. C., Lewis, G. F., Irwin, M. J., et al. 2005, MNRAS, 362, 475

Conn, B. C., Lane, R. R., Lewis, G. F., et al. 2007, MNRAS, 376, 939

Conn, B. C., Lane, R. R., Lewis, G. F., et al. 2008, MNRAS, 390, 1388

de Jong, J. T. A., Butler, D. J., Rix, H.-W., Dolphin, A. E., \& Martínez-Delgado, D. 2007, ApJ, 662, 259

Gilmore, G., \& Reid, N. 1983, MNRAS, 202, 1025

Hammersley, P. L., Garzón, F., Mahoney, T., \& Calbet, X. 1995, MNRAS, 273, 206

Ibata, R. A., Gilmore, G., \& Irwin, M. J. 1994, Nature, 370, 194

Ivezić, Z., Sesar, B., Jurić, M., et al. 2008, ApJ, 684, 287

Kalberla, P. M. W., Dedes, L., Kerp, J., \& Haud, U. 2007, A\&A, 469, 511

López-Corredoira, M. 2006, MNRAS, 369, 1911

López-Corredoira, M., Cabrera-Lavers, A., Garzón, F., \& Hammersley, P. L. 2002, A\&A, 394, 883

López-Corredoira, M., Momany, Y., Zaggia, S., \& Cabrera-Lavers, A. 2007, A\&A, 472, L47

López-Corredoira, M., \& Betancort-Rijo, J. 2009, A\&A, 493, L9

Martin, N. F., Ibata, R. A., Bellazzini, M., et al. 2004, MNRAS, 348, 12

Martínez-Delgado, D., Butler, D. J., Rix, H.-W., et al. 2005, ApJ, 633, 205

Momany, Y., Zaggia, S. R., Bonifacio, P., et al. 2004, A\&A, 421, L29

Momany, Y., Zaggia, S. R., Gilmore, G., et al. 2006, A\&A, 451, 515

Newberg, H. J., Yanny, B., Rockosi, C., et al. 2002, ApJ, 569, 245

Robin, A. C., Creze, M., \& Mohan, V. 1992, ApJ, 400, L25

Robin, A. C., Reylé, C., Derrière, S., \& Picaud, S. 2003, A\&A, 409, 523

Rocha-Pinto, H. J., Majewski, S. R., Skrutskie, M. F., \& Crane, J. D. 2003, ApJ, 594, L115

Schlegel, D. J., Finkbeiner, D. P., \& Davis, M. 1998, ApJ, 500, 525 